\title{
Direct kinetic assay of interactions between small peptides and immobilized antibodies using a surface plasmon resonance biosensor
}

\author{
Paula Gomes ${ }^{a}$, David Andreu ${ }^{\mathrm{b}, *}$ \\ ${ }^{a}$ Centro de Investigação em Química (CIQUP), R. Campo Alegre, 687, P-4169-007 Oporto, Portugal \\ ${ }^{\mathrm{b}}$ Department of Organic Chemistry, University of Barcelona, Martí i Franquès 1, E-08028 Barcelona, Spain
}

Received 10 July 2001; accepted 9 August 2001

\begin{abstract}
A surface plasmon resonance (SPR) protocol is described for the direct kinetic analysis of small antigenic peptides interacting with immobilized monoclonal antibodies (mAb). High peptide concentrations (up to $2.5 \mu \mathrm{M}$ ) and medium mAb surface densities (about $1.5 \mathrm{ng} / \mathrm{mm}^{2}$ ) are needed to ensure measurable binding levels, and fast buffer flow rates (60 $\mu \mathrm{l} / \mathrm{min}$ ) are required to minimize diffusion-controlled kinetics. Good reproducibility levels in the kinetic constants are obtained under these analysis conditions (standard deviations below 10\% of the mean values). Application of this protocol to determine the antigenic ranking of viral peptides shows an excellent agreement between SPR and previous competition enzyme-linked immunosorbent assays (ELISA) on the same peptide/antibody systems. C) 2002 Elsevier Science B.V. All rights reserved.
\end{abstract}

Keywords: Antigen-antibody interactions; Real-time biospecific interaction analysis kinetics; Surface plasmon resonance analysis of small analytes

Abbreviations: $C_{\mathrm{A}}$, analyte concentration; EDC, $N$-ethyl- $N^{\prime}-$ dimethylaminopropylcarbodiimide; ELISA, enzyme-linked immunosorbent assay; Fab, antigen-binding fragment of an antibody; FMDV, foot-and-mouth disease virus; HEPES, $\mathrm{N}$-2-hydroxyethylpiperazine- $N^{\prime}$-2-ethanesulfonic acid; $\mathrm{IC}_{50}, 50 \%$ inhibition concentration; $k_{\mathrm{a}}$, association rate constant $\left(\mathrm{M}^{-1} \mathrm{~s}^{-1}\right) ; K_{\mathrm{A}}$, association thermodynamic constant $\left(\mathrm{M}^{-1}\right) ; k_{\mathrm{d}}$, dissociation rate constant $\left(\mathrm{s}^{-1}\right) ; K_{\mathrm{D}}$, dissociation thermodynamic constant $(\mathrm{M}) ; k_{\mathrm{s}}$, apparent rate constant $\left(\mathrm{s}^{-1}\right)$; mAb, monoclonal antibody; NHS, $N$-hydroxysuccinimide; PBS, phosphate buffer saline; $R$, SPR response at time $t(\mathrm{RU}) ; R_{\text {eq }}$, equilibrium response (RU); RI, bulk refractive index (RU); $R_{\max }$, maximum response (RU); $R_{\text {tot }}$, total SPR response (RU); RU, resonance units; SDS, sodium dodecylsulfate; SPR, surface plasmon resonance; $t_{\text {on }}$, run start time.

* Corresponding author. Tel./fax: +34-93-402-1260.

E-mail address: andreu@qo.ub.es (D. Andreu).

\section{Type of research}

The use of SPR biosensors (Fägerstam et al., 1992; Malmqvist and Karlsson, 1997; Homola et al., 1999) for interaction analysis has made it possible to obtain affinity and kinetic data for a large number of antigen-antibody (Brigham-Burke et al., 1992; VanCott et al., 1994; Oddie et al., 1997; England et al., 1997; Houshmand et al., 1999), protein-protein (Wu et al., 1995), protein-peptide (Lessard et al., 1996) and protein-DNA (Cheskis and Freedman, 1996) systems. Other relevant applications are epitope mapping (Dubs et al., 1992; Saunal and Van Regenmortel, 1995) or selective 
concentration analysis of bioactive molecules in complex samples (Richalet-Sécordel et al., 1997). The majority of the direct single-step SPR analyses reported in the literature (Altschuh et al., 1992; Wu et al., 1995; Lessard et al., 1996; Brigham-Burke et al., 1992; Lemmon et al., 1994; Tamamura et al., 1996; Chao et al., 1996; England et al., 1997) involve analytes weighing above $5 \mathrm{kDa}$. Since the SPR response is directly related to changes in mass on the sensing surface, there is an experimental limitation for direct SPR detection of small analytes, which led to a golden rule in SPR: immobilize the smaller binding partner. A clear example of this rule can be found, for instance, in antigen-antibody interaction studies where antigens are immobilized on the sensor surface and the larger antibodies are used as analytes (Altschuh et al., 1992; Zeder-Lutz et al., 1997). When this rule is not suitable for the purposes in view, alternative SPR approaches are employed, such as multistep sandwich (Cheskis and Freedman, 1996; Huyer et al., 1995; Shen et al., 1996; Lookene et al., 1996) or indirect competitive (Lasonder et al., 1994, 1996; Karlsson, 1994; Zeder-Lutz et al., 1995; Nieba et al., 1996) analysis. However, in antigen-antibody interaction studies, the general rule is that a high number of potential antigens (e.g., peptides with key residue substitutions) are to be screened against a small set of specific antibodies. Thus, antibody immobilization has clear practical benefits over peptide immobilization. Comparison between different peptide antigens is meaningful only if they are analyzed under exactly the same conditions (e.g., all injected over the same antibody surface). Moreover, large analytes (e.g., antibodies) are more prone to generate steric hindrance and mass-transport artifacts that affect true kinetic data.

The protocol that we present here is suited for direct single-step surface plasmon resonance (SPR) analysis of small ligand-large receptor interactions, where small peptides are used as analytes (injected in the buffer continuous flow) and monoclonal antibodies (mAb) are immobilized on the SPR sensor chip surface. The protocol has been optimized and validated using foot-and-mouth disease virus (FMDV) peptides and anti-FMDV neutralizing mAb as the binding partners, as described elsewhere (Gomes et al., 2000a,b, 2001a).

\section{Time required}

2.1. Full kinetic analysis of a peptide-antibody interaction

For routine analyses on a previously prepared sensor surface, $2-3 \mathrm{~h}$ will suffice. Considering also ligand immobilization and instrument maintenance procedures, $4-5 \mathrm{~h}$ will be required.

2.2. Immobilization of the antibody on the sensor chip surface

(1) Preconcentration assays: $60 \mathrm{~min}$

(2) Covalent immobilization: $30 \mathrm{~min}$

(3) Testing regeneration conditions: $30 \mathrm{~min}$

\subsection{Binding kinetics assays}

(1) Blank injections (two runs): $20 \mathrm{~min}$

(2) Analyte injections (sample + regeneration): 20 $\min$

\subsection{Data analysis (BIAevaluation software): $60 \mathrm{~min}$}

\subsection{Maintenance}

(1) Priming the system (once a day or each time a sensor chip is changed): $10 \mathrm{~min}$

(2) "Desorb" (once a week: washing the system in harsh conditions): $30 \mathrm{~min}$

(3) Sanitizing the system (once a month): $40 \mathrm{~min}$

(4) Normalizing the signal (once a week or when buffer is changed): $40 \mathrm{~min}$

\section{Materials}

\subsection{Special equipment}

- The protocol has been optimized on a BIAcore 1000 SPR biosensor

- Personal computer working on a Windows environment (Windows '95, '98, 2000 or NT)

- BIACORE control 3.1 software

- BIAevaluation 3.0 software

- BIAsimulation software (optional) 


\subsection{Certified materials for SPR assays}

Certified materials for running SPR assays on the BIAcore $^{\mathrm{TM}}$ biosensors are commercially available (Biosensor, Uppsala, Sweden):

- CM5 sensor chips, certified grade (code BR1000-12, package of three chips) - carboxymethylated dextran matrix, with $a \geq 4000$ RU binding capacity for a $40-\mathrm{kDa}$ protein standard and with user-defined binding specificity.

- HBS-EP running buffer (code BR-1001-88, $6 \times$ $200 \mathrm{ml})-10 \mathrm{mM}$ HEPES ( $N$-2-hydroxyethylpiperazine- $N$ '-2-ethanesulfonic acid) with $0.15 \mathrm{M} \mathrm{NaCl}$, $3.4 \mathrm{mM}$ EDTA and $0.005 \%$ surfactant $\mathrm{P} 20$ at $\mathrm{pH} 7.4$.

- Amine coupling kit (code BR-1000-50, for 50 immobilizations) - $750 \mathrm{mg} N$-ethyl- $N^{\prime}$-(3-dimethylaminopropyl)carbodiimide (EDC), $115 \mathrm{mg} N$-hydroxysuccinimide (NHS), $10.5 \mathrm{ml}$ ethanolamine hydrochloride.

- BIAmaintenance kit (code BR-1002-22, for 6 months normal usage) - solutions of sucrose $(65 \mathrm{ml})$, glycerol $(30 \mathrm{ml})$, SDS $(90 \mathrm{ml})$, glycine $(90 \mathrm{ml})$, diazolidinyl urea with surfactant P20 $(60 \mathrm{ml})$, sodium hypochlorite $(10 \mathrm{ml})$.

- BIAnormalizing solution (code BR-1003-22, 90 $\mathrm{ml}$ )_for normalization of BIACORE probe signal.

\subsection{Solutions for surface regeneration}

The regeneration procedures corresponding to the assays described in the present protocol may, in principle, be carried out using either $50 \mathrm{mM} \mathrm{HCl}$ or $10 \mathrm{mM} \mathrm{NaOH}$. The most common regenerating agents are:

- acids (10-100 mM HCl, $\mathrm{H}_{3} \mathrm{PO}_{4}$ )

- bases $(10-100 \mathrm{mM} \mathrm{NaOH})$

- salts (1-5 mM NaCl)

- detergents $(0.5 \%$ SDS $)$

- denaturants (8 M urea, $6 \mathrm{M}$ guanidine hydrochloride)

\subsection{Monoclonal antibodies}

Purified mAbs in PBS can be used as stock solutions for subsequent dilution in the immobilization buffer. Generally, mAb stock solutions correspond to ca. $20 \mathrm{mg}$ (antibody)/ml (PBS) and are diluted to ca. $5 \mu \mathrm{g} / \mathrm{ml}$ in the chosen immobilization buffer.

\subsection{Immobilization buffers}

Preconcentration assays are performed in order to establish which is the best immobilization buffer. Electrostatic preconcentration is best achieved at low ionic strength. A 10-mM sodium acetate buffer with $\mathrm{pH}=5.5$ is generally adequate for $\mathrm{mAb}$ amine coupling immobilization on a CM5 sensor chip. The most common immobilization buffers for sensor chip CM5 are:

- $10 \mathrm{mM}$ sodium formate $(\mathrm{pH}=3.0-4.5)$

- $10 \mathrm{mM}$ sodium acetate $(\mathrm{pH}=4.0-5.5)$

- $5 \mathrm{mM}$ sodium maleate $(\mathrm{pH}=5.5-6.0)$

\subsection{Peptides}

Peptide $2.5 \mathrm{mM}$ stock solutions in water or 100 $\mathrm{mM}$ acetic acid can be prepared for 1000-fold and subsequent serial dilutions in the SPR running buffer (HBS). Thus, peptide solutions injected on the biosensor typically range from 2500 to $20 \mathrm{nM}$ in HBS.

\section{Detailed procedure}

\subsection{Preparing the system}

System preparation and routine maintenance will not be described in detail since they are presented in the instrumentation manuals. These procedures are almost entirely automated and computer-controlled through interactive software in an icon-based windows environment.

(i) Dock the new sensor chip, replace the HBS running buffer bottle by a fresh one and prime the system.

(ii) Normalize the probe signal according to the manufacturer's instructions.

\subsection{Preconcentration assays}

(iii) Prepare different $\mathrm{mAb}$ solutions to test for the best immobilizing conditions. Different $\mathrm{mAb}$ concentrations (e.g., 5, 10 and $50 \mu \mathrm{g} / \mathrm{ml}$ ) and immobilization buffers (e.g., $10 \mathrm{mM}$ formate, $\mathrm{pH} \mathrm{4.5;10} \mathrm{mM}$ 
acetate, $\mathrm{pH} 5.0 ; 10 \mathrm{mM}$ acetate, $\mathrm{pH}$ 5.5) should be considered.

(iv) Select one out of the four independent CM5 sensor chip flow cells and set the running buffer flow rate to $5 \mu \mathrm{l} / \mathrm{min}$.

(v) Inject sequentially $25 \mu \mathrm{l}$ of each one of the different $\mathrm{mAb}$ solutions prepared in (iii) (5-min injections), with short (1-min) pulses of a 1-M ethanolamine hydrochloride solution $(\mathrm{pH}=8.5)$ between each injection.

(vi) Examine carefully which combination of $\mathrm{mAb}$ concentration/immobilization buffer $\mathrm{pH}$ is most suitable for efficient ligand electrostatic preconcentration on the sensor chip surface. This corresponds to the lowest ligand concentration and to the highest $\mathrm{pH}$ giving maximum response. Immobilization conditions leading to extremely high $\mathrm{mAb}$ attachment rates (steep ascent) should be avoided.

\section{3. mAb immobilization by covalent amine coupling}

Once immobilization conditions are chosen, the mAb can be covalently bound to the sensor chip surface. The amine coupling procedure involves chemical activation of the CM5 surface carboxyl groups and subsequent covalent binding to the $\mathrm{mAb}$ primary amino groups.

(vii) Prepare the activating mixture by mixing 35 $\mu l$ of $0.05 \mathrm{M}$ NHS in water with $35 \mu \mathrm{l}$ of $0.2 \mathrm{M}$ EDC in water (the NHS and EDC solutions must be kept separately below $0{ }^{\circ} \mathrm{C}$ and should be mixed immediately before usage).

(viii) Select the flow cell and set the running buffer flow rate to $5 \mu \mathrm{l} / \mathrm{min}$.

(ix) Inject $35 \mu 1$ ( $7 \mathrm{~min}$ ) of the activating mixture (a response will be observed due to a change in the refractive index).

(x) Immobilize the ligand by injecting $35 \mu \mathrm{l}$ (7 $\mathrm{min}$ ) of the mAb solution chosen in the preconcentration assays, inspecting carefully the slope of the response ascent and the maximum level reached.

(xi) Block the nonreacted surface active sites by injecting $35 \mu \mathrm{l}(7 \mathrm{~min})$ of $1 \mathrm{M}$ ethanolamine hydrochloride adjusted to $\mathrm{pH}$ 8.5. This will also serve to break remaining ligand-surface electrostatic bonds.

(xii) Measure the amount of immobilized ligand by subtracting the initial ("empty" flow cell) from the final baseline level (1000 resonance units-RU
— correspond to a 1-ng $/ \mathrm{mm}^{2}$ ligand surface density). When performing kinetic analyses, the ligand density should be as low as possible, provided signal-to-noise ratios are adequate. Direct detection of small peptide antigens (ca. $1.5 \mathrm{kDa}$ ) binding to immobilized mAbs (ca. $150 \mathrm{kDa}$ ) on a Biacore 1000 generally requires immobilization responses of about 1800 RU.

(xiii) Test the regeneration conditions of the surface: this is done by repeated cycles of analyte injection (e.g, $25 \mu \mathrm{l}$ of a $600-\mathrm{nM}$ solution of the antigenic peptide specific for the immobilized $\mathrm{mAb}$ ) followed by a short pulse (1-3 $\mathrm{min}$ ) of a regenerating solution (the most common ones are mentioned in Section 3.3). A suitable regenerating agent provides full recovery of the baseline level at the end of each cycle while preserving ligand activity (checked by the constancy of analyte binding level in repeated cycles).

\subsection{Binding kinetics assays}

(xiv) Dock the sensor chip containing the immobilized $\mathrm{mAb}$, replace the HBS bottle by a new one, prime the system and normalize the probe signal according to the manufacturer's instructions.

(xv) Prepare the peptide solutions to be injected. Six or seven different analyte concentrations, e.g., a dilution series ranging from 2500 to $20 \mathrm{nM}$ in HBS, will suffice. One blank sample (buffer only) and a negative control analyte (e.g., scrambled peptide) should be included in the analyses. The regeneration solution should also be prepared.

(xvi) Set the running buffer flow rate to 60 $\mu \mathrm{l} / \mathrm{min}$ on the flow cell containing the immobilized ligand (for kinetic analyses, buffer flow rates must be higher than $30 \mu \mathrm{l} / \mathrm{min}$ to avoid diffusion-controlled kinetics).

(xvii) Program the injection cycle: use the "kinject mode," which minimizes sample dispersion and provides user-defined dissociation times in running buffer. Needle-cleaning operations ("predip needle" before analyte injection and "extra clean-up" after regeneration) should be also included in each cycle to avoid carry-over. Each cycle comprises two main steps:

(a) "kinject" $90 \mu \mathrm{l}(1.5 \mathrm{~min})$ of sample solution followed by $4 \mathrm{~min}(240 \mathrm{~s})$ dissociation in running buffer. 
(b) inject $60 \mu \mathrm{l}(1 \mathrm{~min})$ of the regenerating solution.

(xviii) Program the peptide binding assays: each peptide should be analyzed at least at six different concentrations (each corresponding to one injection cycle as described in (xvii)). Each measurement should be run at least in triplicate and injections should preferably follow a random order. Flush the system whenever a new peptide is to be screened and prime the system once a day.

\subsection{Data processing and analysis}

Data processing is done by means of the BIAEvaluation $^{\mathrm{TM}}$ software available from Biosensor. Experimental curves (i.e., sensorgrams) corresponding to the same analyte (at different concentrations) are simultaneously processed. The software includes several kinetic models and nonlinear least squares methods to optimize parameter values. Simple kinetic models perfectly described by integrated rate equations use analytical integration, while more complex ones (e.g., involving mass transport limitations, ligand or analyte heterogeneity, conformational changes, analyte multivalency or ligand cooperativity) use numerical integration.

(xix) Open a new BIAevaluation file and, from there, access all the experimental curves corresponding to a given peptide-mAb system analyzed under identical conditions (except for varying peptide concentration). Also from the same file, open the experimental curves corresponding to the blank run and to the negative-control peptide injections.

(xx) Adjust the time scale (abscissa) so that $t=0$ (injection start) is the same for all curves, and the baseline level (ordinate, before injection start) so that it equals $0 \mathrm{RU}$ in all sensorgrams.

(xxi) Delete the useless parts of the sensorgrams (e.g., the regeneration pulses), after which subtract the blank run (buffer only) curve to all the others (this will eliminate buffer response and instrumental drifts or artifacts).

(xxii) Subtract from each peptide concentration curve the one from the scrambled peptide, to eliminate nonspecific binding.

(xxiii) Fit the set of binding curves by global curve fitting to those kinetic models compatible with your system. Judge which one gives the best fit and the most reliable parameters (a 1:1 Langmuirian behavior-pseudo-first order reaction-should be expected for the interaction between each antigen molecule and each one of the Fabs on the immobilized $\mathrm{mAb}$ ).

The fitting models are based on "blind" mathematical tools and the "best fit" depends on the ability of the fitting algorithm to converge for the true minimum and on the number of parameters that can be varied in the model, i.e., the complexity of the model (O'Shannessy et al., 1993; Morton et al., 1995). Therefore, caution must be taken when judging the "best fit" from a purely mathematical point of view. In general, the best choice is the simplest model of those giving reasonably good fits.

(xxiv) Once the "best fit" is chosen, a further detailed evaluation should be performed in order to establish data consistency (Schuck and Minton, 1996). Different zones of the experimental curves should be used for fitting purposes. Local fittings (each sensorgram separately) should be done and compared with globally fitted data. When applicable, analytical integration methods (separate fitting of association and dissociation phases) should be tested and compared with numerical integration methods. This means that, for a 1:1 interaction (pseudo-first order kinetics), data should be fitted as follows:

(a) global fitting to the $1: 1$ interaction model (numerical integration);

(b) local fitting (each concentration separately) to the 1:1 interaction model (numerical integration);

(c) local fitting, separate $k_{\mathrm{a}} / k_{\mathrm{d}}$ (analytical integration in each one of the separate association and dissociation phases).

If kinetic parameters are consistent throughout all these fits, the kinetic model chosen is most probably correct and interaction data are meaningful.

\section{Results}

In this section, examples of the expected results will be presented for each one of the main stages of the analysis protocols. 


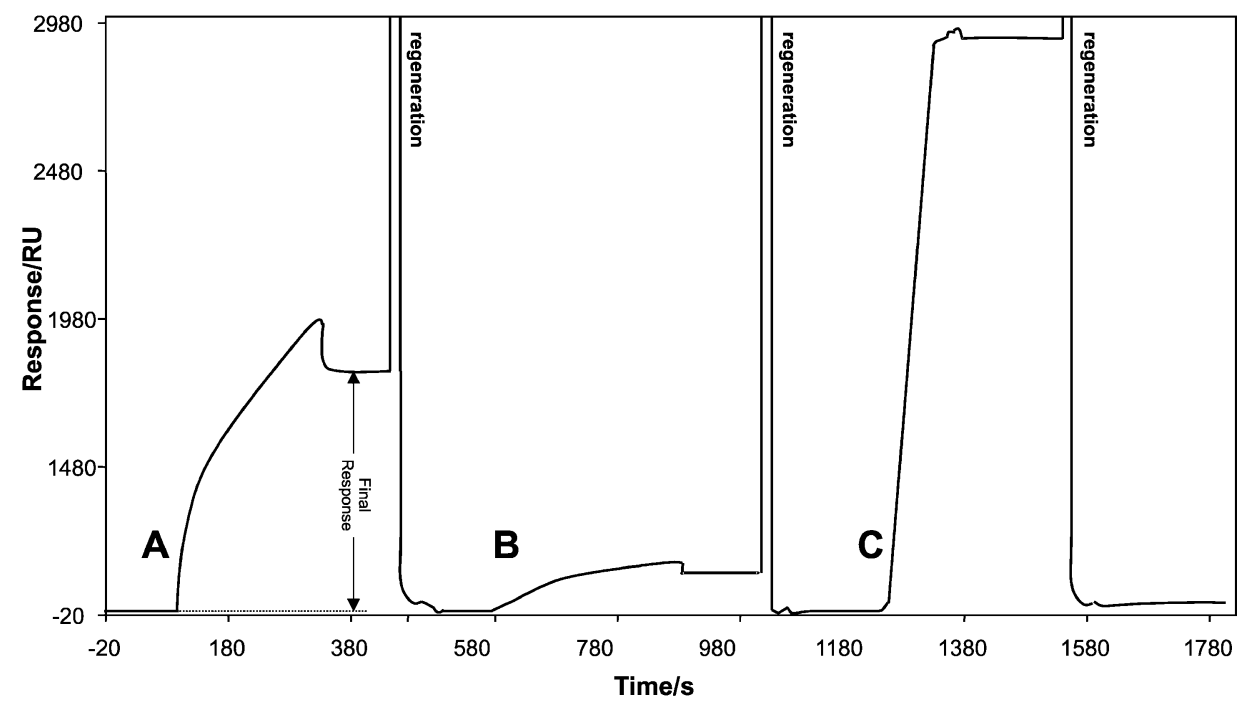

Fig. 1. Example of three successive electrostatic pre-concentration assays. (A) Injection of a $5 \mu \mathrm{g} / \mathrm{ml} \mathrm{mAb}$ solution in $10 \mathrm{mM}$ acetate buffer, $\mathrm{pH}$ 5.5, leads to an efficient mAb preconcentration on the carboxylmethyl-dextran matrix of the sensor chip and to a satisfactory final response level. (B) Injection of a $5 \mu \mathrm{g} / \mathrm{ml} \mathrm{mAb}$ solution in $10 \mathrm{mM}$ formate buffer, $\mathrm{pH} 4.5$, leads to a slow and inefficient electrostatic preconcentration of the ligand. (C) Increasing mAb concentration to $50 \mu \mathrm{g} / \mathrm{ml}$ in $10 \mathrm{mM}$ acetate buffer, $\mathrm{pH} 5.5$, leads to a fast preconcentration and to a too high final response.
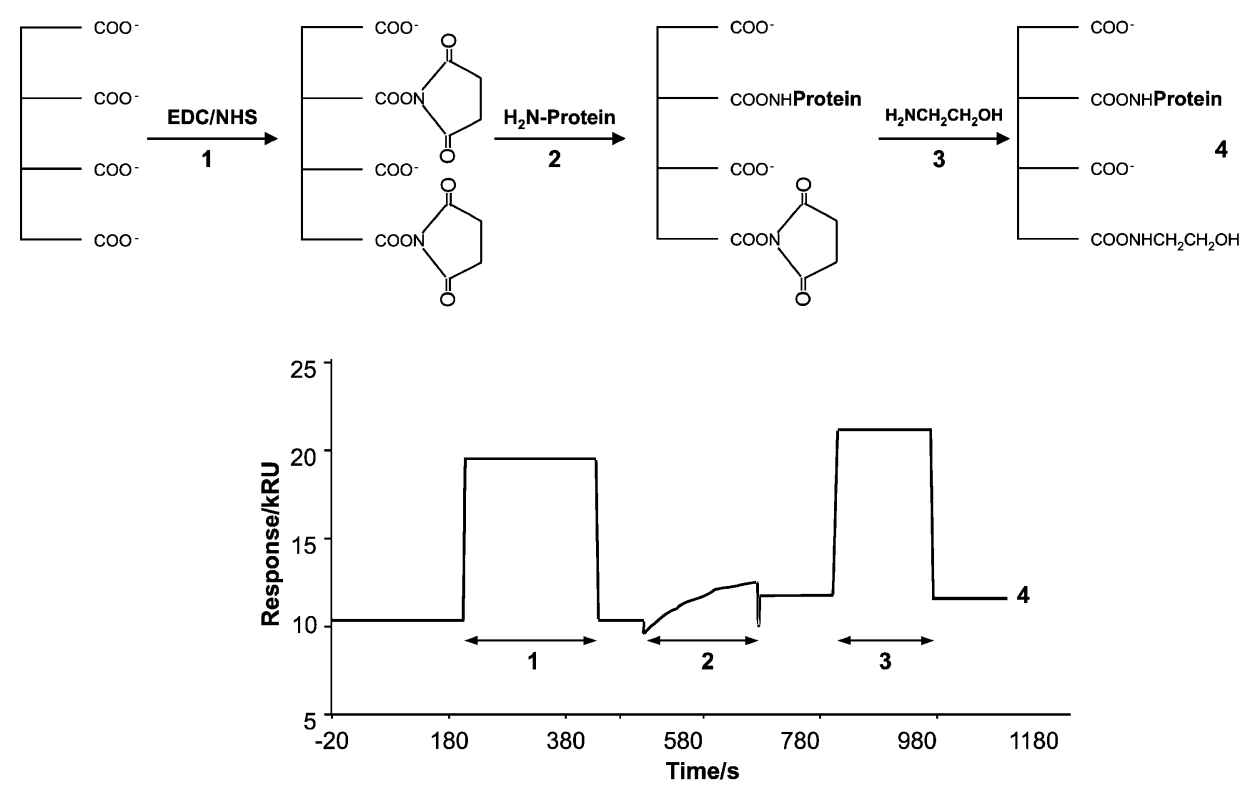

Fig. 2. Example of a covalent immobilization of antibody on a CM5 sensor chip. (1) The carboxyl groups are reacted with a NHS/EDC mixture and reactive NHS esters are formed. (2) The antibody solution is injected and coupling reaction (through the primary amino groups from the antibody lysine residues) is allowed to proceed. (3) Remaining reactive NHS ester sites are blocked with ethanolamine hydrochloride ( $\mathrm{pH}$ 8.5). (4) The final antibody surface is ready. 


\subsection{Preconcentration assays}

Fig. 1 illustrates the results of three sequential preconcentration assays. Using ca. $1700 \mathrm{RU}$ as a reasonable immobilization level for the direct kinetic assay of small peptide binding to an antibody surface, situation A $(5 \mu \mathrm{g} / \mathrm{ml} \mathrm{mAb}$ in $10 \mathrm{mM}$ acetate buffer, $\mathrm{pH} 5.5)$ is clearly the most satisfactory. In B $(5 \mu \mathrm{g} / \mathrm{ml} \mathrm{mAb}$ in $10 \mathrm{mM}$ formate buffer, $\mathrm{pH} 4.5)$, $\mathrm{mAb}$ response increases rather slowly and the final $\mathrm{mAb}$ level is insufficient. In contrast, situation C (50 $\mu \mathrm{g} / \mathrm{ml} \mathrm{mAb}$ in $10 \mathrm{mM}$ acetate buffer, $\mathrm{pH} 5.5)$ corresponds to a fast mAb uptake by the surface resulting in a too high $\mathrm{mAb}$ final density.

\subsection{Antibody covalent immobilization}

A standard ligand covalent immobilization monitored by SPR is depicted in Fig. 2. In a first stage
(1), the EDC/NHS activating mixture is injected with the consequent increase in the SPR signal due to a change in the bulk refractive index. The $\mathrm{mAb}$ solution is then injected and the binding event (2) can be followed in real time. Once the adequate binding level is reached, the remaining active carboxyl-NHS esters are blocked with ethanolamine hydrochloride (3), causing a significant change in the bulk refractive index. The biospecific mAb surface is then ready to be used (4).

\subsection{Binding assays}

The binding assays consist of sequential peptide injection plus regeneration cycles. Fig. 3A shows the three main stages observed when monitoring the biospecific interaction in real time: (1) Analyte binding to the immobilized ligand (association). (2) Bound analyte detaching from the immobilized lig-
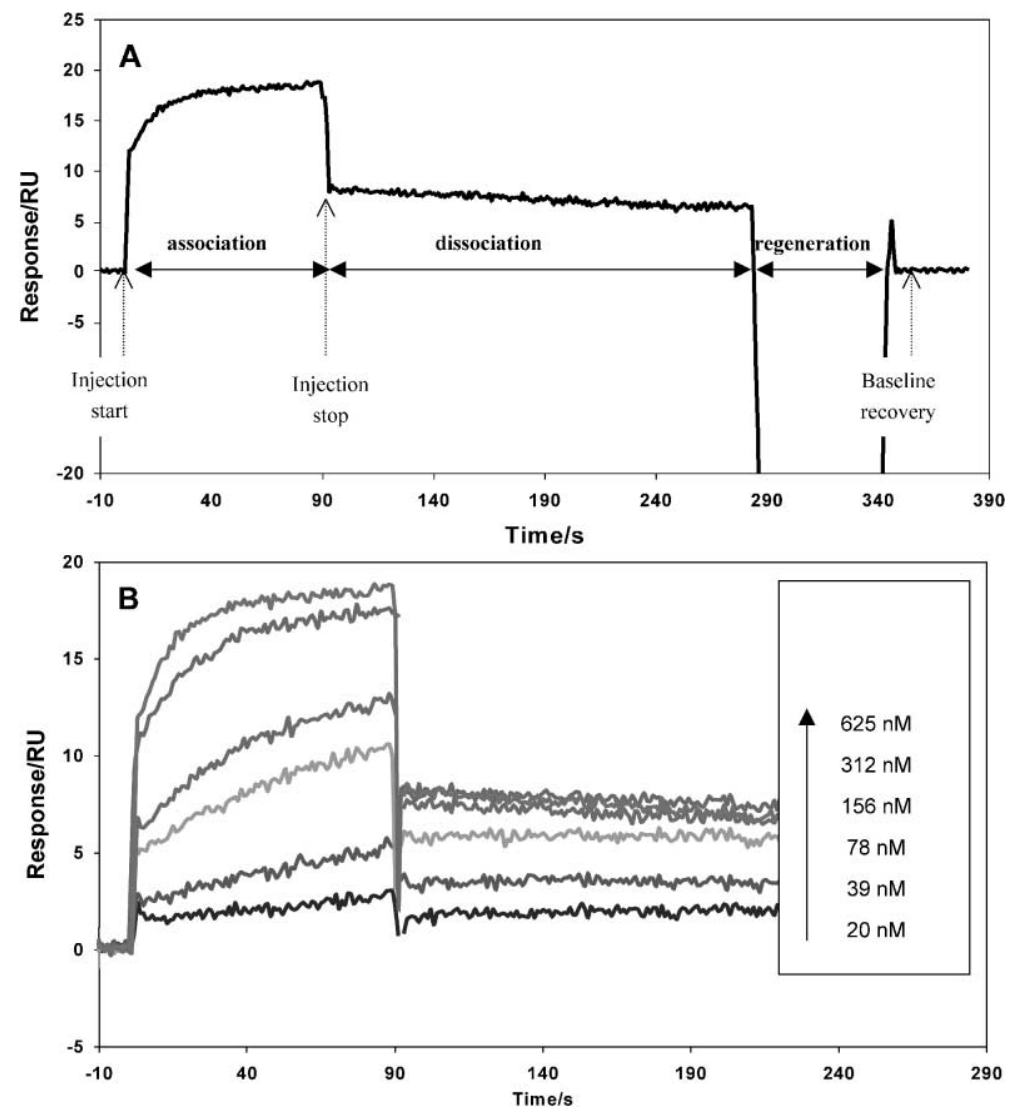

Fig. 3. (A) One injection cycle. (B) Superposition of several binding curves (sensorgrams) corresponding to distinct injection cycles (different concentrations of the same peptide). 
and (dissociation in running buffer). (3) Ligand surface regeneration.

Each cycle corresponds to a new sample, so that all blanks, controls, different peptide concentrations and assay repeats are covered. When a full set (i.e., all concentrations of a given peptide) of injection cycles is finished, the corresponding sensorgrams can be transformed in order to eliminate irrelevant regions (e.g., regeneration pulses) and to normalize the time and response axes. This results in the superposition of several sensorgrams (Fig. 3B), ready to be processed by the curve fitting software.
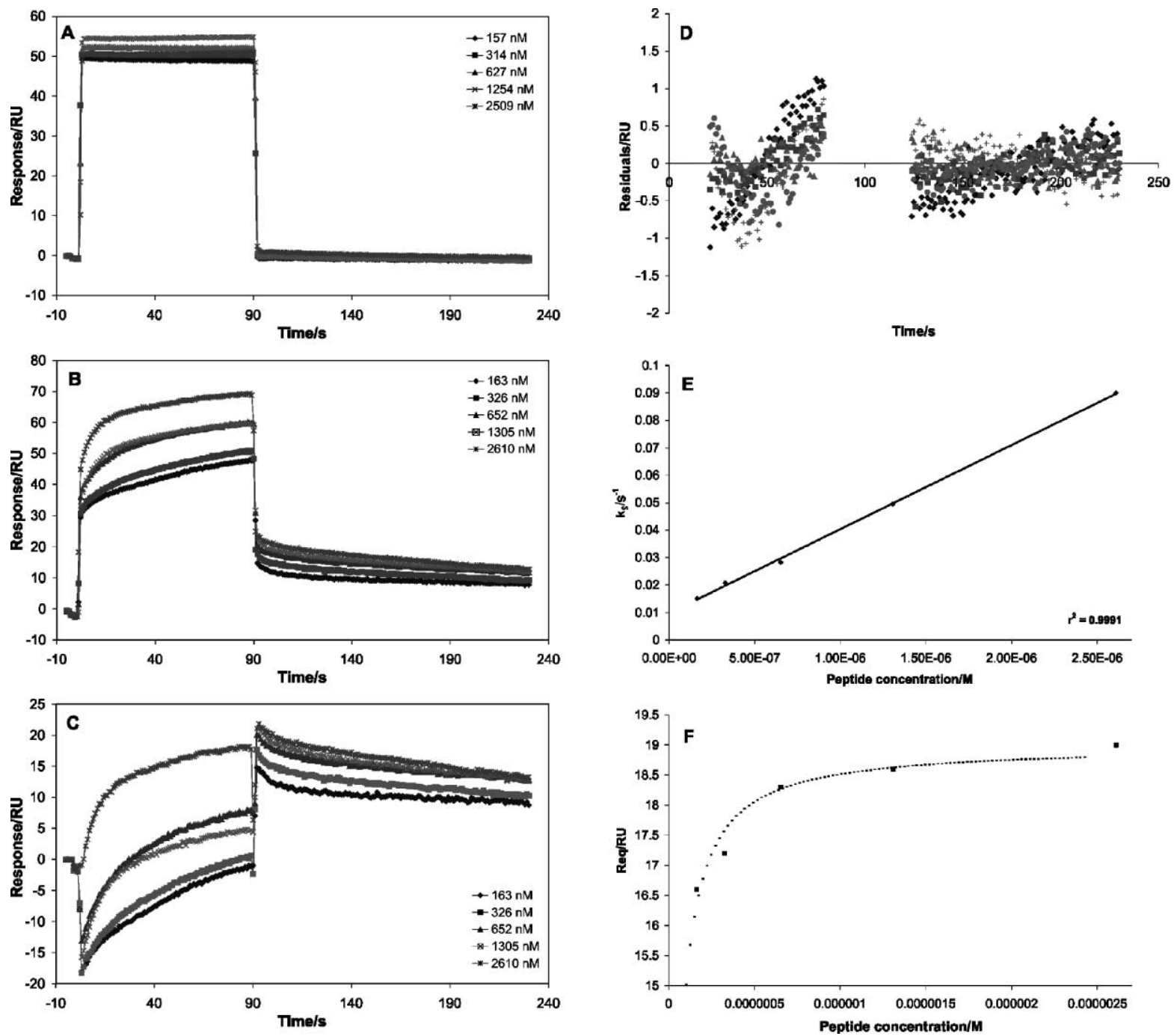

Fig. 4. Illustration of the different stages in the analysis of the interaction between an FMDV peptide and an anti-FMDV neutralizing mAb. (A) Sensorgrams generated by injection of five distinct concentrations of a nonspecific peptide (scrambled sequence) on an anti-FMDV mAb surface. (B) Sensorgrams generated by injection of five distinct concentrations of a specific FMDV peptide on the same anti-FMDV mAb surface. (C) Corrected sensorgrams for the specific FMDV peptide-mAb interactions, obtained by subtraction of curves shown in (A) from the curves shown in (B). (D) Residual data distribution for the association and dissociation phases, after global curve fitting to the 1:1 bimolecular interaction model (numerical integration). (E) Linear correlation between the analyte concentration, $C$, and the apparent rate constant, $k_{\mathrm{s}}$, calculated by local curve fitting to the 1:1 bimolecular interaction model (analytical integration). (F) Correlation between fitted equilibrium response, $R_{\text {eq }}$, and analyte concentration, $C$. 
Table 1

Kinetic and affinity data from the SPR analysis of the peptide-immobilized antibody interaction illustrated in Fig. 4

\begin{tabular}{lclll}
\hline Curve fitting & [Peptide] $(\mathrm{nM})$ & $k_{\mathrm{a}}\left(\mathrm{M}^{-1} \mathrm{~s}^{-1}\right)$ & $k_{\mathrm{d}}\left(\mathrm{s}^{-1}\right)$ & $K_{\mathrm{A}}\left(\mathrm{M}^{-1}\right)$ \\
\hline Global & - & $6.2 \times 10^{4}$ & $2.6 \times 10^{-3}$ & $2.3 \times 10^{7}$ \\
Local, simultaneous $k_{\mathrm{a}} / k_{\mathrm{d}}$ & 152 & $6.0 \times 10^{4}$ & $2.4 \times 10^{-3}$ & $2.5 \times 10^{7}$ \\
& 305 & $5.8 \times 10^{4}$ & $2.6 \times 10^{-3}$ & $2.3 \times 10^{7}$ \\
& 610 & $5.9 \times 10^{4}$ & $2.6 \times 10^{-3}$ & $2.3 \times 10^{7}$ \\
& 1220 & $6.1 \times 10^{4}$ & $2.7 \times 10^{-3}$ & $2.3 \times 10^{7}$ \\
Local, separate $k_{\mathrm{a}} / k_{\mathrm{d}}$ & 2440 & $6.2 \times 10^{4}$ & $2.9 \times 10^{-3}$ & $2.1 \times 10^{7}$ \\
& 152 & $6.7 \times 10^{4}$ & $2.4 \times 10^{-3}$ & $2.8 \times 10^{7}$ \\
& 305 & $6.2 \times 10^{4}$ & $2.6 \times 10^{-3}$ & $2.4 \times 10^{7}$ \\
& 610 & $5.5 \times 10^{4}$ & $2.6 \times 10^{-3}$ & $2.1 \times 10^{7}$ \\
& 1220 & $5.8 \times 10^{4}$ & $2.8 \times 10^{-3}$ & $2.1 \times 10^{7}$ \\
& 2440 & $5.9 \times 10^{4}$ & $2.7 \times 10^{-3}$ & $2.2 \times 10^{7}$ \\
\hline
\end{tabular}

Three different curve fitting methods were tested in order to evaluate the consistency of the fitted parameters.

\subsection{Data processing and evaluation}

Fig. 4 depicts the most important stages in data processing and evaluation. In A, sensorgrams corresponding to a nonspecific peptide injected on a $\mathrm{mAb}$ surface are shown. The sensorgrams are square-wave shaped due to a mere refractive index jump, which is confirmed by the fact that no peptide is bound to the $\mathrm{mAb}$ at the beginning of the dissociation phase. In $\mathrm{B}$, sensorgrams correspond to a specific interaction between an injected peptide and the immobilized $\mathrm{mAb}$. This same interaction is depicted in $\mathrm{C}$, after being corrected by subtraction of the sensorgrams corresponding to the nonspecific peptide analogue (shown in A). Sensorgrams in C were globally fitted to a 1:1 interaction model, with calculated curves totally coincident with the experimental ones and residuals randomly distributed around zero (Fig. 4D), corresponding to a chi-squared lower than 1 . The kinetic parameters obtained are shown in Table 1. These sensorgrams were also fitted locally to the same kinetic model (Table 1).

Further local fitting was performed using the separate $k_{\mathrm{a}} / k_{\mathrm{d}}$ model (Table 1) and the locally fitted apparent rate constant, $k_{\mathrm{s}}$, was plotted against peptide to check the linearity $\left(k_{\mathrm{s}}=k_{\mathrm{a}} \times C+k_{\mathrm{d}}\right)$ expected for a 1:1 interaction kinetics (Fig. 4E). The locally fitted response at equilibrium, $R_{\text {eq }}$, was also plotted against peptide concentration so that the affinity constant $\left(K_{\mathrm{A}}\right)$ values withdrawn from this plot and calculated by the $k_{\mathrm{a}} / k_{\mathrm{d}}$ ratio could be compared (Table 1).

\section{Discussion}

\subsection{Trouble-shooting}

\subsubsection{Immobilization is not satisfactory}

The immobilization level depends on several factors, such as ligand concentration, $\mathrm{pH}$, ionic strength, activation time (EDC/NHS mixture) and injection time (ligand). Generally, lower ligand binding levels can be reached by decreasing ligand concentration, $\mathrm{pH}$, activation and contact times or by increasing ionic strength. Conversely, higher concentrations and activation or contact times, as well as lower ionic strength, contribute to increase ligand immobilization levels.

\subsubsection{Baseline responses increase over repeated cycles}

The regeneration step is not efficient and bound analyte is not fully washed off after each binding cycle. Regenerating agents must be tested and a cocktail approach (Andersson et al., 1999a,b) may be required.

\subsubsection{Binding levels decrease over repeated cycles}

There is loss of ligand activity, either due to ligand inactivation under the analysis conditions em- 
ployed (inadequate buffers, regeneration agents, etc.) or to blockage of ligand binding sites (extremely strong analyte-ligand interactions, ineffective regeneration steps). Different immobilization methodologies or regeneration conditions may solve the problem.

\subsubsection{Expected binding is not observed}

If analyte or ligand degradation prior to usage in the biosensor experiments can be discarded, then ligand inactivation during immobilization must be suspected. Alternative immobilization strategies should be tested (e.g., thiol coupling, binding of biotinylated ligand to a streptavidin surface, immobilization of mAb to an anti-mouse Fc antibody surface).

\subsubsection{Data do not fit to the expected kinetic model}

Generally, antigen-antibody (Fab) interactions display a Langmuirian behavior on the biosensor. Deviations from pseudo-first order kinetics, one of the most difficult problems to solve in biosensor analysis (Morton et al., 1995; O'Shannessy and Winzor, 1996; Hall et al., 1996), can arise from several factors. The consideration is that, when kinetic studies are to be carried out, mass transport effects must be minimized. This can be achieved by decreasing the ligand immobilization level (e.g., to the minimum amount giving a satisfactory signal-to-noise ratio), or by increasing buffer flow rate (always higher than $30 \mu \mathrm{l} / \mathrm{min}$ and as high as sample consumption, thus, permits), or by increasing analyte concentration (as long as surface binding capacity is not saturated). Mass transport influence can be tested by analyzing the effect of different buffer flow rates on analyte initial binding rates (curve slopes at the initial stage of the association step). Another precaution aimed to eliminate mass transport effects in complex dissociation consists in using a ligand solution instead of buffer during the dissociation phase.

Other common sources of deviation are ligand or analyte heterogeneity. The first is mainly due to random immobilization procedures and can be minimized by lowering binding levels or using oriented methodologies such as streptavidin-biotin or anti$\mathrm{Fc}-\mathrm{Fc}$ indirect immobilization. Analyte heterogeneity can be reduced through additional sample purification steps.
The sources of deviation most difficult to deal with are those intrinsic to the binding partners or phenomena, such as analyte multivalency, avidity, or complex binding mechanisms (e.g., involving conformational changes). When these effects are present, the only way to take them into account is to use the more complex fitting models included in the evaluation software, although it may be difficult to judge whether a good fit corresponds to the real binding mechanism (Schuck, 1997).

\subsubsection{Buffer and sample refractive indices mismatch}

Whenever sample and running buffers are different, nonspecific bulk refractive index (RI) jumps take place (square-wave shaped signals superimpose to the binding curves). Although such bulk RI response may be eliminated by subtraction of a blank run, useful information from stages immediately after the injection pulse may be lost. Thus, sample buffer should resemble the running buffer as close as possible.

\subsubsection{Nonspecific binding}

Nonspecific binding may become a problem when using unpurified samples, such as cell lysates, hybridomas, etc. Anyway, nonspecific binding should be checked by one of the following ways. (1) Sample injection on both the specific cell and a reference cell. This reference cell must be prepared as similarly as possible to the specific one (e.g., same coupling chemistry to immobilize a similar amount of inactivated ligand). (2) Another approach, perhaps more appropriate, consists of injection of a nonspecific analyte (e.g., peptide with randomized sequence).

BIAcore 2000 and 3000 instruments allow to monitor interactions on the four different sensor chip cells with a single sample injection, thus, providing simultaneous analysis of analyte binding to three different receptors plus a reference cell at minimal sample costs.

\subsection{Alternative procedures}

The direct single-step approach presented here is the simplest way to study biospecific interactions and is advisable for kinetic studies. However, sometimes the systems under study cannot be suitably 
characterized by this method and alternative approaches may be required. Standard alternative SPR methodologies include the following:

\subsubsection{Direct multistep approach}

This consists of immobilization of a ligand followed by binding of a specific analyte followed by injection of a second binding partner (that binds the first analyte) is injected. Each binding stage is monitored in real-time and this approach is often employed for binding site analysis (Dubs et al., 1992) and analyte response enhancement (Van Regenmortel et al., 1994).

\subsubsection{Indirect surface-competition assay}

This is used in kinetic studies of low molecular weight analytes and consists of injecting a specific high molecular weight analyte followed by competition experiments using the small target analyte as competitor (Karlsson, 1994). This requires a macromolecule possessing the same binding specificity of the small target analytes (e.g., a viral protein competing with a small peptide antigen).

\subsubsection{Solution affinity experiments}

These are widely employed for small analyte detection, with the disadvantage that they do not provide kinetic information. This approach resembles a competition ELISA experiment in the sense that a suitable analyte (e.g., native peptide antigen) is immobilized on the sensor surface and preincubated mixtures of analyte-receptor (e.g., other peptide antigens + specific antibody) are injected. Incubating variable analyte concentrations with a constant receptor concentration allows to build inhibition curves (i.e., free receptor concentration vs. analyte concentration), from which binding constants can be withdrawn (Nieba et al., 1996; Gomes et al., 2001b,c,d).

\section{Essential references}

\subsection{Original papers}

- Fägerstam, L.G., Frostell-Karlsson, A., Karlsson, R., Persson, B. and Rönnberg, I. (1992)

- Hall, D.R., Cann, J.R. and Winzor, D.J. (1996)

- Karlsson, R. (1994)

- Löfas, S. and Johnsson, B. (1990)
- Morton, T.A., Myszka, D. and Chaiken, I. (1995)

- O’Shannessy, D.J. and Winzor, D.J. (1996)

- O'Shannessy, D.J., Brigham-Burke, M. and Peck, K. (1992)

- O'Shannessy, D.J., Brigham-Burke, M., Soneson, K.K., Hensley, P. and Brooks, I. (1993)

\subsection{Review papers}

- Garland, P.B. (1996)

- Schuck, P. (1997)

- Homola, J., Yee, S.S. and Gauglitz, G. (1999)

\subsection{Other sources}

- "Biacore instrument handbook," Biosensor (1994)

- "BIAevaluation software handbook: version 3.0," Biosensor (1997)

- "BIAplications handbook," Biosensor (1994)

- http://www.biacore.com

\section{Quick procedure}

(i) Prepare peptide stock solutions, ca. $2.5 \mathrm{mM}$ in $0.1 \mathrm{M}$ acetic acid, and quantitate by amino acid analysis.

(ii) Prepare $\mathrm{mAb}$ stock solutions, ca. $15 \mathrm{mg} / \mathrm{ml}$ in PBS, and quantitate by measuring optical density at $280 \mathrm{~nm}$ [considering $1 \mathrm{OD}_{280} \approx 0.75 \mathrm{mg}$ (protein) $/ \mathrm{ml}$.

(iii) Prepare $\mathrm{mAb}$ solutions for preconcentration assays: start with four to six different solutions, at different $\mathrm{pH}$ and $\mathrm{mAb}$ concentrations. For instance,

- 5, 10 and $50 \mu \mathrm{g} / \mathrm{ml}$ in $10 \mathrm{mM}$ sodium maleate buffer, $\mathrm{pH} 4.5$;

- 5,10 and $50 \mu \mathrm{g} / \mathrm{ml}$ in $10 \mathrm{mM}$ sodium acetate buffer, $\mathrm{pH}$ 5.0;

- 5,10 and $50 \mu \mathrm{g} / \mathrm{ml}$ in $10 \mathrm{mM}$ sodium acetate buffer, pH 5.5 .

(iv) Prepare a 1-M ethanolamine hydrochloride solution, adjusting the $\mathrm{pH}$ to 8.5 (also available from Biosensor). 
(v) Set the biosensor instrument ready, according to the manufacturer's instructions manual:

- replace the HBS running buffer (available from Biosensor) bottle for a new one;

- dock a new sensor chip (BIAcertified CM5 sensor chip) and prime the system;

- normalize the instrument signal, using the BIAnormalizing solution.

(vi) Select one out the four flow cells on the sensor chip and set the buffer flow rate to $5 \mu \mathrm{l} / \mathrm{min}$.

(vii) Program an alternate series of $25-\mu 1$ injections, corresponding to the different $\mathrm{mAb}$ solutions prepared in (iii), and 5- $\mu$ l injections of the $1 \mathrm{M}$ ethanolamine hydrochloride prepared in (iv).

(viii) Decide whether it is necessary to improve preconcentration levels by adjusting mAb solution parameters (concentration, $\mathrm{pH}$, ionic strength). If improvement is required, repeat steps (iii)-(vii). If not, choose the mAb solution giving the best results and follow steps (ix) and (xx) below, for covalent immobilization.

(ix) Prepare 0.2 M EDC and 0.05 M NHS solutions (also available from Biosensor; these solutions, once prepared, should be divided into $100-\mu 1$ aliquots and stored below $0{ }^{\circ} \mathrm{C}$ ).

(x) Mix $50 \mu \mathrm{l}$ of the EDC solution with an equal volume of the NHS solution, and immediately inject $35 \mu \mathrm{l}$ of this mixture at $5 \mu \mathrm{l} / \mathrm{min}$, to activate the sensor surface. Solutions can be mixed using the automatic sampling unit of the instrument.

(xi) Inject $35 \mu 1$ of the mAb solution chosen in (viii). Binding levels can be controlled at this stage by either interrupting the injection or appending extra injections of the mAb solution.

(xii) Block the remaining active sites on the surface with a $30-\mu$ linjection of $1 \mathrm{M}$ ethanolamine hydrochloride, $\mathrm{pH} 8.5$.

(xiii) Prepare solutions for testing regeneration conditions, starting with the most commonly used for $\mathrm{mAb}$-peptide binding assays:

- $10 \mathrm{mM} \mathrm{HCl}$;

- $10 \mathrm{mM} \mathrm{NaOH}$.

(xiv) Select a peptide expected to bind significantly to the mAb (e.g., the native antigenic se- quence) and dilute the stock solution to $600 \mathrm{nM}$ in HBS.

(xv) Test the regenerating agents by alternating injections of $15 \mu \mathrm{l}$ of peptide and $10 \mu \mathrm{l}$ of regenerating solutions. If common regenerating agents are not adequate, try other possibilities until an agent capable of restoring the baseline level while keeping $\mathrm{mAb}$ binding activity is found. Then, proceed to the binding kinetics analyses as described below.

(xvi) Prepare a peptide dilution series: 1000-fold and further serial dilutions of stock solution in HBS, covering six to eight different peptide concentrations (e.g., 2500-20 nM) . Include the nonspecific peptide dilution series and a blank sample (HBS only).

(xvii) Replace the HBS bottle by a new one, prime the system and normalize the signal as in (v).

(xviii) Set the buffer flow rate to $60 \mu \mathrm{l} / \mathrm{min}$.

(xix) Program the injection cycle (relevant operational commands shown in italics):

- predip needle in HBS;

- kinject $90 \mu \mathrm{l}$ of peptide solution plus $240 \mathrm{~s}$ dissociation in running buffer;

- inject $60 \mu \mathrm{l}$ of the regenerating solution;

- extracleanup needle.

Each injection cycle corresponds to a single analyte sample. Programmed cycles must cover all peptide concentrations plus nonspecific peptide samples and blank runs. Flush the system whenever peptide is changed.

(xx) Prepare raw data obtained in (xix) for processing with the BIAevaluation software: group in the same file all binding curves (sensorgrams) corresponding to the same peptide (different concentrations), to the nonspecific peptide and to the blank runs.

(xxi) Following manufacturer's instructions, normalize all sensorgrams by setting all baseline levels to $0 \mathrm{RU}$ (response units) and all injection starts to $0 \mathrm{~s}$.

(xxii) Delete irrelevant parts of the sensorgrams, such as the regeneration pulses, spikes and alike.

(xxiii) Transform the sensorgrams by subtracting the blank run response.

(xxiv) Further correction of the experimental data is done by subtraction of the nonspecific peptide response (for a given concentration) from the spe- 
cific peptide binding curve (for the same concentration).

(xxv) Select the corrected peptide binding curves and fit them globally to the simplest kinetic model (1:1 bimolecular interaction), choosing binding curve regions as wide as possible so that injection pulses and bulk RI jumps are avoided.

(xxvi) Fit sensorgrams locally (each binding curve separately) to test for kinetic data consistency.

(xxvii) Repeat sensorgram local fitting, now considering association and dissociation steps separately, as a further test for data consistency. Check for linearity of $k_{\mathrm{s}}=f(\mathrm{Conc})$.

(xviii) Judge on fitting model suitableness and kinetic data reliability. If results are satisfactory, proceed to the next peptide. If not, other kinetic models can be tested or, most probably, the experimental setup must be changed (starting with mAb immobilization levels and peptide concentration range).

\section{References}

Altschuh, D., Dubs, M.C., Weiss, E., Zeder-Lutz, G., Van Regenmortel, M.H.V., 1992. Determination of kinetic constants for the interaction between monoclonal antibody and peptides using surface plasmon resonance. Biochemistry 31, 62986304.

Andersson, K., Areskoug, D., Hardenborg, E., 1999a. Exploring buffer space for molecular interactions. J. Mol. Recognit. 12, 36-43.

Andersson, K., Hamalainen, M., Malmqvist, M., 1999b. Identification and optimization of regeneration conditions for affinity-based biosensor assays. A multivariate cocktail approach. Anal. Chem. 71, 2475-2481.

Brigham-Burke, M., Edwards, J.R., O'Shannessy, D.J., 1992. Detection of receptor-ligand interactions using surface plasmon resonance: model studies employing the HIV-1 gp120/ CD4 interaction. Anal. Biochem. 205, 125-131.

Chao, H., Houston, M.E., Grothe, S., Kay, C.M., O’Connor-McCourt, M., Irvin, R.T., Hodges, R.S., 1996. Kinetic study on the formation of a de novo designed heterodimeric coiled-coil: use of surface plasmon resonance to monitor the association and dissociation of polypeptide chains. Biochemistry 35, 12175-12185.

Cheskis, B., Freedman, L.P., 1996. Modulation of nuclear receptor interactions by ligands: kinetic analysis using surface plasmon resonance. Biochemistry 35, 3309-3318.

Dubs, M.C., Altschuh, D., Van Regenmortel, M.H.V., 1992. Mapping of viral epitopes with conformationally specific monoclonal antibodies using biosensor technology. J. Chromatogr. 597, 391-396.
England, P., Brégère, F., Bedouelle, H., 1997. Energetic and kinetic contributions of contact residues of antibody D1.3 in the interaction with lysozyme. Biochemistry 36, 164-172.

Fägerstam, L., Frostell-Karlsson, ̊., Karlsson, R., Persson, B., Rönnberg, I., 1992. Biospecific interaction analysis using surface plasmon resonance detection applied to kinetic, binding site and concentration analysis. J. Chromatogr. 597, 397-410.

Gomes, P., Giralt, E., Andreu, D., 2000a. Surface plasmon resonance screening of synthetic peptides mimicking the immunodominant region of $\mathrm{C}-\mathrm{S} 8 \mathrm{c} 1$ foot-and-mouth disease virus. Vaccine 18, 362-370.

Gomes, P., Giralt, E., Andreu, D., 2000b. Direct single-step surface plasmon resonance analysis of interactions between small peptides and immobilized monoclonal antibodies. J. Immunol. Methods 235, 101-111.

Gomes, P., Giralt, E., Andreu, D., 2001a. Molecular analysis of peptides from the $\mathrm{GH}$ loop of foot-and-mouth disease virus C-S30 using surface plasmon resonance: a role for kinetic rate constants. Mol. Immunol. 37, 975-985.

Gomes, P., Giralt, E., Andreu, D., 2001b. Antigenicity modulation upon peptide cyclization: application to the GH loop of footand-mouth disease virus strain $\mathrm{C}_{1}$-Barcelona. Vaccine 9, 3459-3466.

Gomes, P., Giralt, E., Andreu, D., 2001. Submitted for publication.

Gomes, P., Giralt, E., Andreu, D., 2001. In preparation.

Hall, D.R., Cann, J.R., Winzor, D.J., 1996. Demonstration of an upper limit to the range of association rate constants amenable to study by biosensor technology based on surface plasmon resonance. Anal. Biochem. 235, 175-184.

Homola, J., Yee, S.S., Gauglitz, G., 1999. Surface plasmon resonance sensors: review. Sens. Actuators, B 54, 3-15.

Houshmand, H., Fröman, G., Magnusson, G., 1999. Use of bacteriophage $\mathrm{T} 7$ displayed peptides for determination of monoclonal antibody specificity and biosensor analysis of the binding reaction. Anal. Biochem. 240, 209-214.

Huyer, G., Li, Z.M., Adam, M., Huckle, W.R., Ramachandran, C., 1995. Direct determination of the sequence recognition requirements of the $\mathrm{SH} 2$ domains of SH-PTP2. Biochemistry 34, 1040-1049.

Karlsson, R., 1994. Real-time competitive kinetic analysis of interactions between low-molecular-weight ligands in solution and surface-immobilized receptors. Anal. Biochem. 221, 142 151.

Lasonder, E., Bloemhoff, W., Welling, G.W., 1994. Interaction of lysozyme with synthetic anti-lysozyme D1.3 antibody fragments studied by affinity chromatography and surface plasmon resonance. J. Chromatogr., A 676, 91-98.

Lasonder, E., Schellekens, G.A., Koedjik, D.G.A.M., Damhof, R.A., Welling-Wester, S., Fejilbrrief, M., Scheffer, A.J., Welling, G.W., 1996. Kinetic analysis of synthetic analogues of linear-epitope peptides of glycoprotein D of herpes simplex virus type I by surface plasmon resonance. Eur. J. Biochem. 240, 209-214.

Lemmon, M.A., Ladbury, J.E., Mandiyan, V., Zhou, M., Schlessinger, J., 1994. Independent binding of peptide ligands to the SH2 and SH3 domains of Grb2. J. Biol. Chem. 269, 31653-31658. 
Lessard, I.A.D., Fuller, C., Perhm, R.N., 1996. Competitive interaction of component enzymes with the peripheral subunit-binding domain of the pyruvate-dehydrogenase multienzyme complex of Baccilus stearothermophilus: kinetic analysis using surface plasmon resonance detection. Biochemistry 35, 16863-16870.

Lookene, A., Chevreuil, O., Østergaard, P., Olivecrona, G., 1996. Interaction of lipoprotein lipase with heparin fragments and with heparan sulfate: stoichiometry, stabilization and kinetics. Biochemistry 35, 12155-12163.

Malmqvist, M., Karlsson, R., 1997. Biomolecular interaction analysis: affinity biosensor technologies for functional analysis of proteins. Curr. Opin. Chem. Biol. 1, 378-383.

Morton, T., Myszka, D., Chaiken, I., 1995. Interpreting complex binding kinetics from optical biosensors: a comparison of analysis by linearization, the integrated rate equation and numerical integration. Anal. Biochem. 227, 176-185.

Nieba, L., Krebber, A., Plükthun, A., 1996. Competition BIAcore for measuring true affinities: large differences from values determined from binding kinetics. Anal. Biochem. 234, 155165.

Oddie, G.W., Gruen, L.C., Odgers, G.A., King, L.G., Kortt, A.A., 1997. Identification and minimization of nonideal binding effects in BIAcore analysis: ferritin/anti-ferritin Fab' interaction as a model system. Anal. Biochem. 253, 103-111.

O'Shannessy, D.J., Winzor, D.J., 1996. Interpretation of deviations from pseudo-first order kinetic behavior in the characterization of ligand binding by biosensor technology. Anal. Biochem. 236, 275-283.

O’Shannessy, D.J., Brigham-Burke, M., Soneson, K.K., Hensley, P., Brooks, I., 1993. Determination of rate and equilibrium binding constants for macromolecular interactions using surface plasmon resonance: use of nonlinear least squares methods. Anal. Biochem. 212, 457-468.

Richalet-Sécordel, P.M., Rauffer-Bruyere, N., Christensen, L.L., Ofenloch-Haehnle, B., Seidel, C., Van Regenmortel, M.H.V., 1997. Concentration measurement of unpurified proteins using biosensor technology under conditions of partial mass transport limitation. Anal. Biochem. 249, 165-173.
Saunal, H., Van Regenmortel, M.H.V., 1995. Mapping of viral conformational epitopes using biosensor measurements. J. Immunol. Methods 183, 33-41.

Schuck, P., 1997. Use of surface plasmon resonance to probe the equilibrium and dynamic aspects of interactions between biological macromolecules. Annu. Rev. Biophys. Biomol. Struct. 26, 541-566.

Schuck, P., Minton, A.P., 1996. Kinetic analysis of biosensor data: elementary tests for auto-consistency. Trends Rev. Biochem. Sci. 21, 458-460.

Shen, B.J., Hage, T., Sebald, W., 1996. Global and local determinants for the kinetics of interleukin-4/interleukin-4 receptor $\alpha$ chain interaction: a biosensor study employing recombinant interleukin-4 binding protein. Eur. J. Biochem. 240, 252-261.

Tamamura, H., Otaka, A., Murakami, T., Ishihara, T., Ibuka, T., Waki, M., Matsumoto, A., Yamamoto, N., Fujii, N., 1996. Interaction of an anti-HIV peptide, T22, with gp120 and CD4. Biochem. Biophys. Res. Commun. 219, 555-559.

Van Regenmortel, M.H.V., Altschuh, D., Pellequer, J.L., RichaletSécordel, P., Saunal, H., Wiley, J.A., Zeder-Lutz, G., 1994. Analysis of viral antigens using biosensor technology. Methods: Comp. Methods Enzymol. 6, 177-197.

VanCott, T.C., Bethcke, F.R., Polonis, V.R., Gorny, M.K., ZollaPazner, S., Redfield, R.R., Birx, D.L., 1994. Dissociation rate of antibody-gp120 binding interactions is predictive of V3mediated neutralization of HIV-1. J. Immunol. 153, 449-458.

Wu, Z., Johnsson, K., Choi, Y., Ciardelli, T.L., 1995. Ligand binding analysis of soluble interleukin 2-receptor complexes by surface plasmon resonance. J. Biol. Chem. 270, 1604516051.

Zeder-Lutz, G., Rauffer, N., Altschuh, D., Van Regenmortel, M.H.V., 1995. Analysis of cyclosporin interactions with antibodies and cyclophilin using BIAcore. J. Immunol. Methods $183,131-140$

Zeder-Lutz, G., Zuber, E., Witz, J., Van Regenmortel, M.H.V., 1997. Thermodynamic analysis of antigen-antibody binding using biosensor measurements at different temperatures. Anal. Biochem. 246, 123-132. 